\title{
Unrestricted Fibonacci and Lucas Quaternions
}

\author{
Ahmet Daşdemir ${ }^{1 *}$ and Göksal Bilgici ${ }^{2}$ \\ ${ }^{1}$ Department of Mathematics, Faculty of Arts and Sciences, Kastamonu University, Kastamonu, Turkey \\ ${ }^{2}$ Department of Elementary Mathematics Education, Faculty of Education, Kastamonu University, Kastamonu, Turkey \\ * Corresponding author
}

\section{Article Info}

Keywords: Binet's formula, Catalan's identity, Fibonacci quaternion, Generating function, Lucas quaternion 2010 AMS: 11B39, 15B36, 11R52

Received: 14 June 2020

Accepted: 20 January 2021

Available online: 29 January 2021

\begin{abstract}
Many quaternion numbers associated with Fibonacci and Lucas numbers or even their generalizations have been defined and widely discussed so far. In all the studies, the coefficients of these quaternions have been selected from consecutive terms of these numbers. In this study, we define other generalizations for the usual Fibonacci and Lucas quaternions. We also present some properties, including the Binet's formulas and d'Ocagne's identities, for these types of quaternions.
\end{abstract}

\section{Introduction}

Since Sir William Rowan Hamilton introduced the quaternion algebra in 1843, their usage areas have developed rapidly. Due to the fact that the quaternions are encountered in many problems from elastodynamics, quantum mechanics, elasticity theory and many other fields of modern sciences, they have been studied widely.

A quaternion $q$ can be regarded as a quadruple of real numbers and is formally defined by

$$
q=q_{0}+i q_{1}+j q_{2}+k q_{3}
$$

where $q_{0}, q_{1}, q_{2}$, and $q_{3}$ are any real numbers and the standard basis $\{1, i, j, k\}$ satisfies

$$
i^{2}=j^{2}=k^{2}=-1, i j=-j i=k, j k=-k j=i, k i=-i k=j
$$

The conjugate of $q$ is

$$
q^{*}=q_{0}-i q_{1}-j q_{2}-k q_{3}
$$

and, the norm of $q$ is

Email addresses and ORCID numbers: ahmetdasdemir37@gmail.com, https://orcid.org/0000-0001-8352-2020 (A. Daşdemir), gbilgici@kastamonu.edu.tr, https://orcid.org/0000-0001-9964-5578 (G. Bilgici) 


$$
N(q)=q q^{*}=q_{0}^{2}+q_{1}^{2}+q_{2}^{2}+q_{3}^{2}
$$

Fibonacci numbers are recursively defined as

$$
F_{n}=F_{n-1}+F_{n-2},
$$

with the initial terms $F_{0}=0$ and $F_{1}=1$. The Lucas numbers satisfy the same recurrence relation but with the initial terms $L_{0}=2$ and $L_{1}=1$. Binet's formulas for the Fibonacci and Lucas numbers are

$$
F_{n}=\frac{\alpha^{n}-\beta^{n}}{\alpha-\beta} \text { and } L_{n}=\alpha^{n}+\beta^{n}
$$

respectively. Here, $\alpha=\frac{1+\sqrt{5}}{2}$ and $\beta=\frac{1-\sqrt{5}}{2}$ are the positive and negative roots of $x^{2}-x-1=0$, respectively.

Horadam [1] defined Fibonacci quaternions as

$$
Q_{n}:=F_{n}+i F_{n+1}+j F_{n+2}+k F_{n+3},
$$

where $F_{n}$ is the $n$th term of the Fibonacci sequence. Iyer [2] gave a similar definition for Lucas quaternions by the relation

$$
T_{n}:=L_{n}+i L_{n+1}+j L_{n+2}+k L_{n+3},
$$

and gave their many properties, where $L_{n}$ is the $n$th Lucas number.

Halici [3] gave Binet's formulas for the Fibonacci and Lucas quaternions as follows:

$$
Q_{n}=\frac{\alpha}{\alpha-\underline{\beta} \beta} \text { and } T_{n}=\underline{\alpha} \alpha+\underline{\beta} \beta
$$

where $\underline{\alpha}=1+i \alpha+j \alpha^{2}+k \alpha^{3}$ and $\underline{\beta}=1+i \beta+j \beta^{2}+k \beta^{3}$.

There exist many papers devoted to generalizations of the quaternion sequences in (1.1) and (1.2) today. For example, the references in [4]-[11] can be investigated. Note that all authors have used a known generalization of Fibonacci and Lucas numbers and have placed these numbers in coefficients of the basis vectors similar to the format given by Horadam and Iyer. In this study, we present an another perspective to the Fibonacci and Lucas quaternions. According to our approach, we define a new classes of quaternions whose the coefficients are arbitrarily selected from these splendid integers. The outline of this paper is as follows: In Section 2, we introduce the unrestricted Fibonacci and Lucas quaternions and give Binet's formulas and the generating functions for these quaternions. Furthermore, we present certain special identities such as d'Ocagne's identity and Catalan's identity; in Section 3, we display many fundamental properties and some sum formulas for these quaternion families.

\section{Main results}

Here, we present our definitions, some concepts and results. First of all, we give a definition in the following.

Definition 2.1. Let $p, r$ and $s$ be arbitrary integers. Hence, nth unrestricted Fibonacci and Lucas quaternions are given by the relations

$$
\mathscr{F}_{n}^{(p, r, s)}:=F_{n}+i F_{n+p}+j F_{n+r}+k F_{n+s}
$$

and

$$
\mathscr{L}_{n}^{(p, r, s)}:=L_{n}+i L_{n+p}+j L_{n+r}+k L_{n+s}
$$

respectively. 
According to our definitions, we have the following special cases:

- For $p=r=s=-n$, the usual Fibonacci numbers are obtained:

$$
\mathscr{F}_{n}^{(-n,-n,-n)}=F_{n}
$$

- For $p=1$ and $r=s=-n$, the Gaussian Fibonacci numbers are obtained:

$$
\mathscr{F}_{n}^{(1,-n,-n)}=F_{n}+i F_{n+1}
$$

- For $p=1, r=2$ and $s=3$, the well-known Fibonacci and Lucas quaternions are obtained:

$$
\begin{aligned}
& \mathscr{F}_{n}^{(1,2,3)}=Q_{n}=F_{n}+i F_{n+1}+j F_{n+2}+k F_{n+3} \\
& \mathscr{L}_{n}^{(1,2,3)}=T_{n}=L_{n}+i L_{n+1}+j L_{n+2}+k L_{n+3}
\end{aligned}
$$

Taking Eqs. (2.1) and (2.2) into account, we directly obtain

$$
\mathscr{F}_{n}^{(p, r, s)}=\mathscr{F}_{n-1}^{(p, r, s)}+\mathscr{F}_{n-2}^{(p, r, s)}
$$

and

$$
\mathscr{L}_{n}^{(p, r, s)}=\mathscr{L}_{n-1}^{(p, r, s)}+\mathscr{L}_{n-2}^{(p, r, s)}
$$

by the recurrence relations of Fibonacci and Lucas numbers respectively.

To make it easier to present and prove the results of the rest of paper, we now present next theorem that states Binet's formulas for the unrestricted Fibonacci and Lucas quaternions.

Theorem 2.2. (Binet's Formula) Let $n$ be an integer. Then the Binet's formulas of the unrestricted Fibonacci and Lucas quaternions are

$$
\mathscr{F}_{n}^{(p, r, s)}=\frac{\breve{\alpha} \alpha^{n}-\breve{\beta} \beta^{n}}{\alpha-\beta}
$$

and

$$
\mathscr{L}_{n}^{(p, r, s)}=\breve{\alpha} \alpha^{n}+\breve{\beta} \beta^{n}
$$

where $\breve{\alpha}=1+i \alpha^{p}+j \alpha^{r}+k \alpha^{s}$ and $\breve{\beta}=1+i \beta^{p}+j \beta^{r}+k \beta^{s}$.

Proof. Applying Binet's formulas of the classic Fibonacci numbers to the definition of the unrestricted Fibonacci quaternions, we obtain

$$
\begin{aligned}
\mathscr{F}_{n}^{(p, r, s)} & =F_{n}+i F_{n+p}+j F_{n+r}+k F_{n+s} \\
& =\frac{1}{\alpha-\beta}\left(\alpha^{n}-\beta^{n}+i\left(\alpha^{n+p}-\beta^{n+p}\right)+j\left(\alpha^{n+r}-\beta^{n+r}\right)+k\left(\alpha^{n+s}-\beta^{n+s}\right)\right) \\
& =\frac{1}{\alpha-\beta}\left(\alpha^{n}\left(1+i \alpha^{p}+j \alpha^{r}+k \alpha^{s}\right)-\beta^{n}\left(1+i \beta^{p}+j \beta^{r}+k \beta^{s}\right)\right),
\end{aligned}
$$

and the last equation gives Eq. (2.5). The other can be proved similarly. 


\begin{tabular}{|c|c|c|c|c|}
\hline$\cdot$ & $\breve{\alpha}$ & $\breve{\beta}$ & $\breve{\alpha}^{*}$ & $\breve{\beta}^{*}$ \\
\hline$\breve{\alpha}$ & $2 \breve{\alpha}-N(\breve{\alpha})$ & $B+\sqrt{5} C$ & $N(\breve{\alpha})$ & $A-\sqrt{5} C+2 \gamma$ \\
\hline$\breve{\beta}$ & $B-\sqrt{5} C$ & $2 \breve{\beta}-N(\breve{\beta})$ & $-A+\sqrt{5} C$ & $N(\breve{\beta})$ \\
\hline$\breve{\alpha}^{*}$ & $N(\breve{\alpha})$ & $-A-\sqrt{5} C$ & $2 \breve{\alpha}^{*}-N(\breve{\alpha})$ & $-B+\sqrt{5} C-2 \gamma+4$ \\
\hline$\breve{\beta}^{*}$ & $A+\sqrt{5} C+2 \gamma$ & $N(\breve{\beta})$ & $-B-\sqrt{5} C-2 \gamma+4$ & $2 \breve{\beta}^{*}-N(\breve{\beta})$ \\
\hline
\end{tabular}

Table 1: The multiplicative properties of $\breve{\alpha}$ and $\breve{\beta}$

Table 1 displays important features corresponding to the multiplication of $\breve{\alpha}$ and $\breve{\beta}$. They will undertake important tasks in the process of proofing the next theorems. Note that each of them can easily be proven by certain elementary operations and we omit the details. In Table 1, the following notations are used:

$$
\begin{gathered}
A=\sqrt{5} \mathscr{F}_{0}^{(p, r, s)}-\gamma, \\
B=\mathscr{L}_{0}^{(p, r, s)}-\gamma, \\
C=i(-1)^{s} F_{r-s}+j(-1)^{p} F_{s-p}+k(-1)^{r} F_{p-r}
\end{gathered}
$$

and

$$
\gamma=1+(-1)^{p}+(-1)^{r}+(-1)^{s} .
$$

Note that $r-s, s-p$, and $p-r$ may be positive or negative.

The next theorem gives d'Ocagne's identities for the considered quaternions.

Theorem 2.3. (d'Ocagne's identity) Let $m$ and $n$ be any integers. Hence, we have

$$
\mathscr{F}_{m}^{(p, r, s)} \mathscr{F}_{n+1}^{(p, r, s)}-\mathscr{F}_{m+1}^{(p, r, s)} \mathscr{F}_{n}^{(p, r, s)}=(-1)^{n}\left(B F_{m-n}+C L_{m-n}\right)
$$

and

$$
\mathscr{L}_{m}^{(p, r, s)} \mathscr{L}_{n+1}^{(p, r, s)}-\mathscr{L}_{m+1}^{(p, r, s)} \mathscr{L}_{n}^{(p, r, s)}=-5(-1)^{n}\left(B F_{m-n}+C L_{m-n}\right)
$$

Proof. From the Binet's formula in (2.5), we have

$$
\begin{aligned}
\mathscr{F}_{m}^{(p, r, s)} \mathscr{F}_{n+1}^{(p, r, s)}-\mathscr{F}_{m+1}^{(p, r, s)} \mathscr{F}_{n}^{(p, r, s)} & =\frac{1}{5}\left[\left(\breve{\alpha} \alpha^{m}-\breve{\beta} \beta^{m}\right)\left(\breve{\alpha} \alpha^{n+1}-\breve{\beta} \beta^{n+1}\right)-\left(\breve{\alpha} \alpha^{m+1}-\breve{\beta} \beta^{m+1}\right)\left(\breve{\alpha} \alpha^{n}-\breve{\beta} \beta^{n}\right)\right] \\
& =\frac{\sqrt{5}}{5}(-1)^{n}\left(\breve{\alpha} \breve{\beta} \alpha^{m-n}-\breve{\beta} \breve{\alpha} \beta^{m-n}\right)
\end{aligned}
$$

and by Table 1 ,

$$
\begin{aligned}
\mathscr{F}_{m}^{(p, r, s)} \mathscr{F}_{n+1}^{(p, r, s)}-\mathscr{F}_{m+1}^{(p, r, s)} \mathscr{F}_{n}^{(p, r, s)} & =\frac{\sqrt{5}}{5}(-1)^{n}\left[(B+C \sqrt{5}) \alpha^{m-n}-(B-C \sqrt{5}) \beta^{m-n}\right] \\
& =\frac{\sqrt{5}}{5}(-1)^{n}\left[B\left(\alpha^{m-n}-\beta^{m-n}\right)+C \sqrt{5}\left(\alpha^{m-n}+\beta^{m-n}\right)\right] .
\end{aligned}
$$

We obtain the first identity from the last equation. Repeating the same procedure, the second identity can be obtained. 
Now we give Catalan's identities of the unrestricted Fibonacci and Lucas quaternions.

Theorem 2.4. (Catalan's identity) For any integers $m$ and $n$, we have

$$
\mathscr{F}_{m+n}^{(p, r, s)} \mathscr{F}_{m-n}^{(p, r, s)}-\left[\mathscr{F}_{m}^{(p, r, s)}\right]^{2}=(-1)^{m+n+1} F_{n}\left(B F_{n}+C L_{n}\right)
$$

and

$$
\mathscr{L}_{m+n}^{(p, r, s)} \mathscr{L}_{m-n}^{(p, r, s)}-\left[\mathscr{L}_{m}^{(p, r, s)}\right]^{2}=5(-1)^{m+n} F_{n}\left(B F_{n}+C L_{n}\right)
$$

Proof. By Eq. (2.5) and Table 1, we can write

$$
\begin{aligned}
\mathscr{F}_{m+n}^{(p, r, s)} \mathscr{F}_{m-n}^{(p, r, s)}-\left[\mathscr{F}_{m}^{(p, r, s)}\right]^{2} & =\frac{1}{5}\left[\left(\breve{\alpha} \alpha^{m+n}-\breve{\beta} \beta^{m+n}\right)\left(\breve{\alpha} \alpha^{m-n}-\breve{\beta} \beta^{m-n}\right)-\left(\breve{\alpha} \alpha^{m}-\breve{\beta} \beta^{m}\right)^{2}\right] \\
& =\frac{1}{5}\left[\left((-1)^{m-n+1}\left(\breve{\alpha} \breve{\beta} \alpha^{2 n}+\breve{\beta} \breve{\alpha} \beta^{2 n}\right)+(-1)^{m} 2 C\right]\right. \\
& =\frac{1}{5}\left[(-1)^{m+n-1}\left((B+\sqrt{5} C) \alpha^{2 n}+(B-\sqrt{5} C) \beta^{2 n}\right)+(-1)^{m} B\right] \\
& =\frac{1}{5}\left[(-1)^{m+n-1}\left(B\left(\alpha^{2 n}+\beta^{2 n}\right)+5 C\left(\frac{\alpha^{2 n}-\beta^{2 n}}{\alpha-\beta}\right)\right)+(-1)^{m} B\right] \\
& =\frac{1}{5}\left[(-1)^{m+n-1}\left(B L_{2 n}+5 C F_{2 n}\right)+(-1)^{m} B\right] .
\end{aligned}
$$

Substituting the identities $5 F_{n}^{2}=L_{2 n}-(-1)^{n}([12, \mathrm{p} .42])$ and $F_{2 n}=F_{n} L_{n}([12, \mathrm{p} .14])$ give the desired result. Similarly, the second identity can be obtained.

For the case $n=1$ in Theorem 2.4, we attain Cassini's identities, which are given in the following.

Corollary 2.5. (Cassini's identity) For any integer $m$, we have

$$
\mathscr{F}_{m+1}^{(p, r, s)} \mathscr{F}_{m-1}^{(p, r, s)}-\left[\mathscr{F}_{m}^{(p, r, s)}\right]^{2}=(-1)^{m}(B+C)
$$

and

$$
\mathscr{L}_{m+1}^{(p, r, s)} \mathscr{L}_{m-1}^{(p, r, s)}-\left[\mathscr{L}_{m}^{(p, r, s)}\right]^{2}=-5(-1)^{m}(B+C)
$$

Note that the above identities can be re-written for the usual forms of the Fibonacci and Lucas quaternions in the case $(p, r, s)=(1,2,3)$. In this case, we can summarize them as follows:

- D’Ocagne's identities are as follows:

$$
\begin{gathered}
Q_{m} Q_{n+1}-Q_{m+1} Q_{n}=(-1)^{n}\left[T_{0} F_{m-n}+\left(-Q_{0}+3 k\right) L_{m-n}\right] \\
K_{m} K_{n+1}-K_{m+1} K_{n}=-5(-1)^{n}\left[T_{0} F_{m-n}+\left(-Q_{0}+3 k\right) L_{m-n}\right]
\end{gathered}
$$

- Catalan's identities are as follows:

$$
\begin{gathered}
Q_{m+n} Q_{m-n}-Q_{m}^{2}=(-1)^{m} F_{-n}\left[F_{n} T_{0}+\left(-Q_{0}+3 k\right) L_{n}\right] \\
K_{m+n} K_{m-n}-K_{m}^{2}=-5(-1)^{m} F_{-n}\left[F_{n} T_{0}+\left(-Q_{0}+3 k\right) L_{n}\right]
\end{gathered}
$$


- Cassini’s identities are as follows:

$$
\begin{gathered}
Q_{m+1} Q_{m-1}-Q_{m}^{2}=(-1)^{m}\left(Q_{-1}+3 k\right) \\
K_{m+1} K_{m-1}-K_{m}^{2}=-5(-1)^{m}\left(Q_{-1}+3 k\right)
\end{gathered}
$$

The concept of Generating Function is so important research topic since that is helpful tool to solve linear homogeneous recurrence relations with constant coefficients. Here, we investigate both the ordinary generating functions and the exponential generating functions associated with our generalized quaternions. To do this, we introduce the following functions:

$$
\begin{gathered}
G_{\mathscr{F}}(x)=\sum_{n=0}^{\infty} \mathscr{F}_{n}^{(p, r, s)} x^{n}, \\
G_{\mathscr{L}}(x)=\sum_{n=0}^{\infty} \mathscr{L}_{n}^{(p, r, s)} x^{n}, \\
E_{\mathscr{F}}(x)=\sum_{n=0}^{\infty} \mathscr{F}_{n}^{(p, r, s)} \frac{x^{n}}{n !}
\end{gathered}
$$

and

$$
E_{\mathscr{L}}(x)=\sum_{n=0}^{\infty} \mathscr{L}_{n}^{(p, r, s)} \frac{x^{n}}{n !}
$$

Hence, we present another main results of the current paper.

Theorem 2.6. The generating functions for the unrestricted Fibonacci and Lucas quaternions are

$$
G_{\mathscr{F}}(x)=\frac{\mathscr{F}_{0}^{(p, r, s)}+\mathscr{F}_{-1}^{(p, r, s)} x}{1-x-x^{2}}
$$

and

$$
G_{\mathscr{L}}(x)=\frac{\mathscr{L}_{0}^{(p, r, s)}+\mathscr{L}_{-1}^{(p, r, s)} x}{1-x-x^{2}}
$$

Proof. Substituting Eq. (2.3) into Eq. (2.8) and Eq. (2.4) into Eq. (2.9), the results are satisfied. So, this completes the proof.

Theorem 2.7. The exponential generating functions for the unrestricted Fibonacci and Lucas quaternions are

$$
E_{\mathscr{F}}(x)=\frac{\breve{\alpha} e^{\alpha x}-\breve{\beta} e^{\beta x}}{\alpha-\beta}
$$

and

$$
E_{\mathscr{L}}(x)=\breve{\alpha} e^{\alpha x}+\breve{\beta} e^{\beta x}
$$

where e is the famous Euler's number.

Proof. Substituting Eq. (2.5) into Eq. (2.7) leads to

$$
E_{\mathscr{F}}(x)=\sum_{n=0}^{\infty} \mathscr{F}_{n}^{(p, r, s)} \frac{x^{n}}{n !}=\sum_{n=0}^{\infty} \frac{\breve{\alpha} \alpha^{n}-\breve{\beta} \beta^{n}}{\alpha-\beta} \frac{x^{n}}{n !}=\frac{1}{\alpha-\beta}\left(\breve{\alpha} \sum_{n=0}^{\infty} \frac{(\alpha x)^{n}}{n !}-\breve{\beta} \sum_{n=0}^{\infty} \frac{(\beta x)^{n}}{n !}\right)
$$

Considering MacLaurin series of an exponential function, the result follows. The second identity is demonstrated similarly. 


\section{More features}

In this section, we present many properties for the unrestricted Fibonacci and Lucas quaternions and some sum formulas of them. The next theorem present these identities.

Theorem 3.1. Let $m, n$ and $t$ be any integers. Then,

$$
\begin{aligned}
& \mathscr{F}_{n}^{(p+1, r+1, s+1)}=\mathscr{F}_{n}^{(p, r, s)}+\mathscr{F}_{n-1}^{(p, r, s)}-F_{n-1}, \\
& \mathscr{L}_{n}^{(p+1, r+1, s+1)}=\mathscr{L}_{n}^{(p, r, s)}+\mathscr{L}_{n-1}^{(p, r, s)}-L_{n-1}, \\
& \mathscr{F}_{n+1}^{(p, r, s)}=\mathscr{F}_{n}^{(p+1, r+1, s+1)}+F_{n-1}, \\
& \mathscr{L}_{n+1}^{(p, r, s)}=\mathscr{L}_{n}^{(p+1, r+1, s+1)}+L_{n-1}, \\
& \mathscr{L}_{n}^{(p, r, s)}=\mathscr{F}_{n-1}^{(p, r, s)}+\mathscr{F}_{n+1}^{(p, r, s)} \\
& \mathscr{L}_{m+n}^{(p, r, s)} \mathscr{F}_{m+t}^{(p, r, s)}-\mathscr{L}_{m+t}^{(p, r, s)} \mathscr{F}_{m+n}^{(p, r, s)}=2(-1)^{m+n} B \mathscr{F}_{t-n}^{(p, r, s)}, \\
& \mathscr{F}_{m+n}^{(p, r, s)}+(-1)^{n} \mathscr{F}_{m-n}^{(p, r, s)}=\mathscr{F}_{m}^{(p, r, s)} L_{n}, \\
& \mathscr{F}_{m}^{(p, r, s)} \mathscr{L}_{n}^{(p, r, s)}-\mathscr{L}_{n}^{(p, r, s)} \mathscr{F}_{m}^{(p, r, s)}=2(-1)^{m} C L_{n-m} \\
& \mathscr{F}_{m}^{(p, r, s)} \mathscr{L}_{n}^{(p, r, s)}-\mathscr{L}_{m}^{(p, r, s)} \mathscr{F}_{n}^{(p, r, s)}=2(-1)^{n}\left(B F_{m-n}+C L_{m-n}\right), \\
& \mathscr{F}_{n}^{(p, r, s)} \mathscr{F}_{m}^{(p, r, s)}-\mathscr{F}_{m}^{(p, r, s)} \mathscr{F}_{n}^{(p, r, s)}=2(-1)^{m+1} C F_{n-m}, \\
& \mathscr{L}_{n}^{(p, r, s)} \mathscr{L}_{m}^{(p, r, s)}-\mathscr{L}_{m}^{(p, r, s)} \mathscr{L}_{n}^{(p, r, s)}=10(-1)^{m} C F_{n-m}, \\
& \mathscr{F}_{m+n}^{(p, r, s)} \mathscr{F}_{m+n}-\mathscr{F}_{m-n}^{(p, r, s)} \mathscr{F}_{m-n}=\mathscr{F}_{2 m}^{(p, r, s)} F_{2 n}, \\
& \mathscr{L}_{m+n}^{(p, r, s)} L_{m+n}-\mathscr{L}_{m-n}^{(p, r, s)} L_{m-n}=5 \mathscr{F}_{2 m}^{(p, r, s)} F_{2 n}, \\
& \mathscr{F}_{m+n}^{(p, r, s)} L_{m+n}+\mathscr{F}_{m-n}^{(p, r, s)} L_{m-n}=\mathscr{F}_{2 m}^{(p, r, s)} L_{2 n}+2(-1)^{m+n} \mathscr{F}_{0}^{(p, r, s)} \\
& \mathscr{L}_{m+n}^{(p, r, s)} L_{m+n}+\mathscr{L}_{m-n}^{(p, r, s)} L_{m-n}=\mathscr{L}_{2 m}^{(p, r, s)} L_{2 n}+2(-1)^{m+n} \mathscr{L}_{0}^{(p, r, s)} \\
& 5\left[\mathscr{F}_{m}^{(p, r, s)}\right]^{2}-\left[\mathscr{L}_{m}^{(p, r, s)}\right]^{2}=4(-1)^{m+1} B \\
& \mathscr{F}_{m+n}^{(p, r, s)}+(-1)^{n} \mathscr{F}_{m-n}^{(p, r, s)}=\mathscr{F}_{m}^{(p, r, s)} L_{n}, \\
& \mathscr{L}_{m+n}^{(p, r, s)}+(-1)^{n} \mathscr{L}_{m-n}^{(p, r, s)}=\mathscr{L}_{m}^{(p, r, s)} L_{n}
\end{aligned}
$$

and

$$
\mathscr{F}_{2 m}^{(p, r, s)}=F_{m+1} \mathscr{F}_{m}^{(p, r, s)}+F_{m} \mathscr{F}_{m-1}^{(p, r, s)}
$$


Proof. To reduce the volume of the current paper, we give some proofs of the above identities. Since the proofs of the first six identities are done in the same way, we only prove Eq. (3.1). By the definition of unrestricted Fibonacci quaternions, we can write

$$
\begin{aligned}
\mathscr{F}_{n}^{(p+1, r+1, s+1)} & =F_{n}+i F_{n+p+1}+j F_{n+r+1}+k F_{n+s+1} \\
& =F_{n}+i\left(F_{n+p}+F_{n+p-1}\right)+j\left(F_{n+r}+F_{n+r-1}\right)+k\left(F_{n+s}+F_{n+s-1}\right) \\
& =\left(F_{n}+i F_{n+p}+j F_{n+r}+k F_{n+s}\right)+\left(F_{n-1}+i F_{n+p-1}+j F_{n+r-1}+k F_{n+s-1}\right)-F_{n-1} .
\end{aligned}
$$

Other proofs are based on Binet's formulas of the corresponding quaternions. As an example, we show that (3.2) holds. Hence, by employing Eq. (2.6) we obtain

$$
\begin{aligned}
\mathscr{L}_{m+n}^{(p, r, s)} L_{m+n}-\mathscr{L}_{m-n}^{(p, r, s)} L_{m-n} & =\left(\breve{\alpha} \alpha^{m+n}+\breve{\beta} \beta^{m+n}\right)\left(\alpha^{m+n}+\beta^{m+n}\right)-\left(\breve{\alpha} \alpha^{m-n}+\breve{\beta} \beta^{m-n}\right)\left(\alpha^{m-n}+\beta^{m-n}\right) \\
& =\left(\breve{\alpha} \alpha^{2 m+2 n}+\breve{\beta} \beta^{2 m+2 n}-\breve{\alpha} \alpha^{2 m-2 n}-\breve{\beta} \beta^{2 m-2 n}\right) \\
& =\left(\breve{\alpha} \alpha^{2 m+2 n}+\breve{\beta} \beta^{2 m+2 n}-\breve{\alpha} \alpha^{2 m} \beta^{2 n}-\breve{\beta} \alpha^{2 n} \beta^{2 m}\right) \\
& =(\alpha-\beta)^{2}\left(\frac{\breve{\alpha} \alpha^{2 m}-\breve{\beta} \beta^{2 m}}{\alpha-\beta}\right)\left(\frac{\alpha^{2 n}-\beta^{2 n}}{\alpha-\beta}\right) \\
& =5_{2 m}^{(p, r, s)} F_{2 n} .
\end{aligned}
$$

So, the proof is completed.

Now, we list sum formulas for the considered quaternions in the following theorem.

Theorem 3.2. The following summation formulas hold for any integer $n$.

$$
\begin{aligned}
& \sum_{t=0}^{n} \mathscr{F}_{t}^{(p, r, s)}=\mathscr{F}_{n+2}^{(p, r, s)}-\mathscr{F}_{1}^{(p, r, s)} \\
& \sum_{t=0}^{n} \mathscr{L}_{t}^{(p, r, s)}=\mathscr{L}_{n+2}^{(p, r, s)}-\mathscr{L}_{1}^{(p, r, s)} \\
& \sum_{t=0}^{n} \mathscr{F}_{2 t-1}^{(p, r, s)}=\mathscr{F}_{2 n}^{(p, r, s)}-\mathscr{F}_{0}^{(p, r, s)} \\
& \sum_{t=0}^{n} \mathscr{L}_{2 t-1}^{(p, r, s)}=\mathscr{L}_{2 n}^{(p, r, s)}-\mathscr{L}_{0}^{(p, r, s)} \\
& \sum_{t=0}^{n} \mathscr{F}_{2 t}^{(p, r, s)}=\mathscr{F}_{2 n+1}^{(p, r, s)}-\mathscr{F}_{-1}^{(p, r, s)} \\
& \sum_{t=0}^{n}\left(\begin{array}{l}
n \\
t
\end{array}\right) \mathscr{F}_{t}^{(p, r, s)}=\mathscr{F}_{2 n}^{(p, r, s)} \\
& \sum_{t=0}^{n} \mathscr{L}_{2 t}^{(p, r, s)}=\mathscr{L}_{2 n+1}^{(p, r, s)}-\mathscr{L}_{-1}^{(p, r, s)}
\end{aligned}
$$

and

$$
\sum_{t=0}^{n}\left(\begin{array}{l}
n \\
t
\end{array}\right) \mathscr{L}_{t}^{(p, r, s)}=\mathscr{L}_{2 n}^{(p, r, s)}
$$


Proof. We will prove some of the above identities again. Let us consider $a_{t}=\mathscr{F}_{t+2}^{(p, r, s)}-\mathscr{F}_{1}^{(p, r, s)}$. Hence, by the definition of the unrestricted Fibonacci quaternions, we obtain

$$
a_{t}-a_{t-1}=\mathscr{F}_{t}^{(p, r, s)}
$$

Applying the idea of creative telescoping [13] to Eq. (3.3), we conclude

$$
\sum_{t=0}^{n} \mathscr{F}_{t}^{(p, r, s)}=\sum_{t=0}^{n}\left(a_{t}-a_{t-1}\right)=a_{n}-a_{-1}
$$

and since $a_{-1}=0$, Eq. (3.3) is attained. Proceeding as in the previous proof, we can obtain the other identities except last two identities.

Now, substituting Eq. (2.5) to Eq. (3.4) leads to

$$
\sum_{t=0}^{n}\left(\begin{array}{l}
n \\
t
\end{array}\right) \mathscr{F}_{t}^{(p, r, s)}=\sum_{t=0}^{n}\left(\begin{array}{l}
n \\
t
\end{array}\right) \frac{\breve{\alpha} \alpha^{n}-\breve{\beta} \beta^{n}}{\alpha-\beta}=\frac{1}{\alpha-\beta}\left(\breve{\alpha} \sum_{t=0}^{n}\left(\begin{array}{l}
n \\
t
\end{array}\right) \alpha^{n}-\breve{\beta} \sum_{t=0}^{n}\left(\begin{array}{l}
n \\
t
\end{array}\right) \beta^{n}\right)
$$

and considering the formal expression of the Binomial Theorem, we obtain

$$
\sum_{t=0}^{n}\left(\begin{array}{l}
n \\
t
\end{array}\right) \mathscr{F}_{t}^{(p, r, s)}=\frac{\breve{\alpha}(\alpha+1)^{n}-\breve{\beta}(\beta+1)^{n}}{\alpha-\beta}
$$

Using $\alpha^{2}=\alpha+1$ and $\beta^{2}=\beta+1$, the proof of Eq. (3.4) is completed. The last equation can also be found in a similar way.

\section{Conclusions}

In this study, we presented other generalizations for the usual Fibonacci and Lucas quaternions and gave many interesting properties of these definitions. In particular, the Binet's formulas, the generating function, some explicit formulas, and special identities such as d'Ocagne's Identities were obtained. Moreover, the examples regarding the reduced cases for our generalizations, from the quaternion forms to the usual integer sequence ones, were investigated. In addition to those, we considered sum formulas for our generalizations including the even and odd subscripts.

We think that the inferences of the paper can be used in several practical applications in applied sciences, e.g. control and system theory, and neural network, etc.

\section{Acknowledgement}

The authors would like to declare the financial support provided by the Research Fund of Kastamonu University under project number KÜBAP-01/2017-1.

\section{References}

[1] A. F. Horadam, Complex Fibonacci numbers and Fibonacci quaternions, Amer. Math. Monthly, 70(2) (1963), $289-291$.

[2] M. R. Iyer, A note on Fibonacci quaternions, The Fibonacci Quart., 7(2) (1969), 225-229.

[3] S. Halici, On Fibonacci quaternions, Adv. Appl. Clifford Algebr., 22(2) (2012), 321-327.

[4] M. N. S. Swamy, On generalized Fibonacci quaternions, The Fibonacci Quart., 11(5) (1973), 547-550.

[5] C. Flaut, V. Shpakivskyi, On generalized Fibonacci quaternions and Fibonacci-Narayana quaternions, Adv. Appl. Clifford Algebr., 23(3) (2013), $673-688$.

[6] M. Akyigit, H. H. Kosal, M. Tosun, Fibonacci generalized quaternions, Adv. Appl. Clifford Algebr., 24(3) (2014), 631-641.

[7] D. Tasci, F. Yalcin, Fibonacci-p quaternions, Adv. Appl. Clifford Algebr., 25(1) (2015), 245-254.

[8] J. L. Ramirez, Some combinatorial properties of the k-Fibonacci and the k-Lucas quaternions, An. St. Univ. Ovidius Constanta, 23(2) (2015), 201-212

[9] F. Torunbalci Aydin, On the bicomplex k-Fibonacci quaternions, Commun. Adv. Math. Sci., 2(3) (2019), $227-234$.

[10] F. Torunbalci Aydin, Hyperbolic Fibonacci sequence, Univers. J. Math. Appl., 2(2) (2019), 59-64.

[11] M. A. Gungor, A. Cihan, On dual hyperbolic numbers with generalized Fibonacci and Lucas numbers components, Fundam. J. Math. Appl., 2(2) (2019), 162-172.

[12] T. Koshy, Fibonacci and Lucas Numbers with Applications, John Wiley and Sons, New York, 2001

[13] D. Zeilberger, The method of creative telescoping, J. Symbolic Comput., 11(3) (1991), 195-204. 